\title{
On operators satisfying an inequality
}

\section{Salah Mecheri*}

\section{${ }^{\text {*Correspondence: }}$}

mecherisalah@hotmail.com

Department of Mathematics,

College of Science, Taibah

University, P.O. Box 30002,

Al-Madinah-Al-Munawarah, Saudi

Arabia

\begin{abstract}
An operator $T$ is said to be $k$-quasi- $*$-class $A$ if $T^{* k}\left(\left|T^{2}\right|-\left|T^{*}\right|^{2}\right) T^{k} \geq 0$, where $k$ is a natural number. Let $d_{A, B} \in B(B(H))$ denote either the generalized derivation $\delta_{A, B}=L_{A}-R_{B}$ or the elementary operator $\Delta_{A, B}=L_{A} R_{B}-I$, where $L_{A}$ and $R_{B}$ are the left and right multiplication operators defined on $B(H)$ by $L_{A}=A X$ and $R_{B}=X B$ respectively. This article concerns some spectral properties of $k$-quasi-*-class $A$ operators in a Hilbert space, as the property of being hereditarily polaroid. We also establish Weyl-type theorems for $T$ and $d_{A, B}$, where $T$ is a $k$-quasi-*-class $A$ operator and $A, B^{*}$ are also $k$-quasi- $*$-class $A$ operators.

MSC: Primary 47B47; 47A30; 47B20; secondary 47B10
\end{abstract}

Keywords: $k$-quasi-*-class $A$; $*$-paranormal operator; Weyl's spectrum

\section{Introduction and preliminaries}

Let $B(H)$ be the algebra of all bounded linear operators acting on an infinite dimensional separable complex Hilbert space $H$. As an easy extension of normal operators, hyponormal operators have been studied by many mathematicians. Although there are many unsolved interesting problems for hyponormal operators (e.g., the invariant subspace problem), one of recent trends in operator theory is studying natural extensions of hyponormal operators. So, we introduce some of these non-hyponormal operators. Recall [1, 2] that $T \in B(H)$ is called hyponormal if $T^{*} T \geq T T^{*}$, and $\mathrm{T}$ is called paranormal (resp., *-paranormal) if $\left\|T^{2} x\right\| \geq\|T x\|^{2}$ (resp. $\left\|T^{2} x\right\| \geq\left\|T^{*} x\right\|^{2}$ ) for all unit vector $x \in H$. Following [2] and [3], we say that $T \in B(H)$ belongs to the class $A$ if $\left|T^{2}\right| \geq|T|^{2}$. Recently Jeon and Kim [3] have considered the following new class of operators: we say that an operator $T \in B(H)$ belongs to the $*$-class $A$ if $\left|T^{2}\right| \geq\left|T^{*}\right|^{2}$.

For brevity, we shall denote the classes of hyponormal operators, paranormal operators, *-paranormal operators, class $A$ operators, and $*$-class $A$ operators by $\mathcal{H}, \mathcal{P N}, \mathcal{P N} \mathcal{N}^{*}, \mathcal{A}$ and $\mathcal{A}^{*}$ respectively. From [1] and [2], it is well known that

$$
\mathcal{H} \subset \mathcal{A} \subset \mathcal{P N} \text { and } \mathcal{H} \subset \mathcal{A}^{*} \subset \mathcal{P N}^{*}
$$

Recently in [4], the authors have extended $*$-class $A$ operators to quasi- $*$-class $A$ operators. An operator $T \in B(H)$ is said to be quasi-*-class $A$ if $T^{*}\left|T^{2}\right| T \geq T^{*}\left|T^{*}\right|^{2} T$. If we denote this class of operators by $\mathcal{Q} \mathcal{A}^{*}$, then

$$
\mathcal{H} \subset \mathcal{A}^{*} \subset \mathcal{Q} \mathcal{A}^{*}
$$

( 2012 Mecheri; licensee Springer. This is an Open Access article distributed under the terms of the Creative Commons Attribution License (http://creativecommons.org/licenses/by/2.0), which permits unrestricted use, distribution, and reproduction in any medium, provided the original work is properly cited. 
As a further generalization of both $*$-class $A$ operators and quasi-*-class $A$ operators, the author in [5] introduced $k$-quasi- $*$-class $A$ operators. An operator $T$ is called $k$-quasi- $*$ class $A$ if

$$
T^{* k}\left(\left|T^{2}\right|-\left|T^{*}\right|^{2}\right) T^{k} \geq 0,
$$

where $k$ is a natural number. Let $\mathcal{K} \mathcal{Q} \mathcal{A}^{*}$ be the class of $k$-quasi- $*$-class $A$ operators. Thus,

$$
\mathcal{H} \subset \mathcal{A}^{*} \subset \mathcal{Q} \mathcal{A}^{*} \subset \mathcal{K} \mathcal{Q} \mathcal{A}^{*}
$$

The spectral properties of quasi-class $A$ and quasi-*-class $A$ operators have been investigated by many authors in the recent years (a useful survey on the spectral properties of these operators may also be found in [6]); see also [4]. In this paper we extend to $k$-quasi*-class $A$ operators some of these results, for instance the property of being hereditarily polaroid already observed for $*$-paranormal operators and $*$-class $A$ operators defined on Hilbert spaces [5].

The fine structure of the spectrum of paranormal operators for class $A$ operators or *paranormal operators has been studied by several authors, in particular, for these classes of operators, it has been proved that they satisfy Weyl's theorem; see for instance $[7,8]$ for paranormal operators, [9] for algebraically class $A$ operators, in [5] for quasi- $*$-class $A$ operators. In this paper we extend these results by proving that some other variants of Weyl's theorem hold for $k$-quasi- $*$-class $A$ operators; for instance, the so-called property $(w)$ introduced by Rakočević in [10] and studied in [11] and [12]. All Weyl-type theorems are established for $T$ and for $d_{A, B} ; T$ is a $k$-quasi-*-class $A$ operator and $A, B^{*}$ are also $k$-quasi-*-class $A$ operators.

We begin by explaining the relevant terminology. Let $X$ be a complex Banach space. For a bounded linear operator $T \in B(X)$, let $N(T)$ denote the null space and $\operatorname{ran} T$ denote the range of $T$. Let $p:=p(T)$ be the ascent of an operator $T$. (I.e., the smallest non-negative integer $p$ such that $N\left(T^{p}\right)=N\left(T^{p+1}\right)$. If such integer does not exist, we put $p(T)=\infty$.) Analogously, let $q:=q(T)$ be the descent of an operator $T$; i.e., the smallest non-negative integer $q$ such that $\operatorname{ran} T^{q}=\operatorname{ran} T^{q+1}$, and if such integer does not exist, we put $q(T)=\infty$. It is well known that if $p(T)$ and $q(T)$ are both finite, then $p(T)=q(T)$ [13, Proposition 38.3]. Moreover, $0<p(\lambda I-T)=q(\lambda I-T)<\infty$ precisely when $\lambda$ is a pole of the resolvent of $T$; see Proposition 50.2 of Heuser [13]. A bounded operator $T \in B(X)$ is said to be polaroid if every isolated point of the spectrum is a pole of the resolvent. $T \in B(X)$ is said to be hereditarily polaroid if the restriction of $T$ to any closed invariant subspace is polaroid. Let $\sigma_{\mathrm{a}}(T)$ denote the classical approximate point spectrum. $T$ is said to be a-polaroid if every $\lambda \in$ iso $\sigma_{\mathrm{a}}(T)$ is a pole of the resolvent of $T$. Obviously,

$$
\text { Ta-polaroid } \Rightarrow T \text { polaroid. }
$$

In [14] it has been observed that if the dual $T^{\prime}$ has SVEP (respectively, $T$ has SVEP), then two conditions for $T$ of being polaroid or $a$-polaroid (respectively, for $T^{\prime}$ ) are equivalent. The following property has a relevant role in local spectral theory and Fredholm operator theory; see the recent monographs by Laursen and Neumann [15] and [16]. A bounded operator $T \in B(X)$ is said to have the single valued extension property (abbreviated SVEP) 
if, for every open subset $G$ of $\mathbb{C}$ and any analytic function $f: G \rightarrow X$ such that $(T-z) f(z) \equiv$ 0 on $G$, we have $f(z) \equiv 0$ on $G$.

We also have

$$
p(\lambda I-T)<\infty \Rightarrow T \text { has SVEP at } \lambda,
$$

and dually, if $T^{\prime}$ denotes the dual of $T$,

$$
q(\lambda I-T)<\infty \quad \Rightarrow \quad T^{\prime} \text { has SVEP at } \lambda \text {; }
$$

see [16, Theorem 3.8]. In the case of Hilbert space operators, the last implication is still true if we replace $T^{\prime}$ with the Hilbert adjoint $T^{*}$. A bounded operator $T \in B(X)$ is said to have Bishop's property $(\beta)$ if for every open subset $G$ of $\mathbb{C}$ and every sequence $f_{n}: G \rightarrow H$ of $H$-valued analytic functions such that $(T-z) f_{n}(z)$ converges uniformly to 0 in norm on compact subsets of $G, f_{n}(z)$ converges uniformly to 0 in norm on compact subsets of $G$. It is known that the property $(\beta)$ for $T$ entails that $T$ has SVEP; see [15] for details.

\section{Main results}

We begin by the following lemma which is the essence of this paper and it is a structure theorem of a $k$-quasi-*-class $A$ operator $T$.

Lemma 2.1 [5] Let $T \in B(H)$ be a $k$-quasi-*-class $A$ operator, the range of $T^{k}$ be not dense and

$$
T=\left(\begin{array}{cc}
T_{1} & T_{2} \\
0 & T_{3}
\end{array}\right) \quad \text { on } H=\left[\operatorname{ran} T^{k}\right] \oplus \operatorname{ker} T^{* k}
$$

Then $T_{1}$ is a $*$-class $A$ operator, $T_{3}^{k}=0$ and $\sigma(T)=\sigma\left(T_{1}\right) \cup\{0\}$.

As a consequence, we obtain the following corollary.

Corollary 2.1 Let $T \in B(H)$ be a $k$-quasi-*-class $A$ operator. If $T_{1}$ is invertible, then $T$ is similar to a direct sum of a *-class $A$ operator and a nilpotent operator.

Proof Since by assumption $0 \notin \sigma(T)$ we have $\sigma\left(T_{1}\right) \cap \sigma\left(T_{3}\right)=\varnothing$, then there exists an operator $S$ such that $T_{1} S-S T_{3}=T_{2}$ [17]. Hence,

$$
T=\left(\begin{array}{cc}
T_{1} & T_{2} \\
0 & T_{3}
\end{array}\right)=\left(\begin{array}{cc}
I & S \\
0 & I
\end{array}\right)^{-1}\left(\begin{array}{cc}
T_{1} & 0 \\
0 & T_{3}
\end{array}\right)\left(\begin{array}{ll}
I & S \\
0 & I
\end{array}\right) .
$$

Corollary 2.2 Let $T$ be a $k$-quasi-*-class $A$ operator. If $T$ is quasinilpotent, then it must be a nilpotent operator.

Proof Invoking Lemma 2.1, we find $\sigma\left(T_{1}\right)=0$. Since $T_{1}$ is $*$-class $A$, we conclude that $T_{1}=0$ [18]. Since $T_{3}^{k}=0$, a computation shows that

$$
T^{k+1}=\left(\begin{array}{cc}
0 & T_{2} T_{3}^{k} \\
0 & T_{3}^{k+1}
\end{array}\right)=0
$$


Lemma 2.2 [5] Let $M$ be a closed $T$-invariant subspace of $H$. Then the restriction $T_{\mid M}$ of a k-quasi-*-class $A$ operator $T$ to $M$ is a k-quasi-*-class $A$ operator.

Theorem 2.1 [5] Let $T \in B(H)$ be k-quasi-*-class A. Then $T$ satisfies Bishop's property $(\beta)$, the single valued extension property and the Dunford property $(C)$.

Lemma 2.3 Let $T \in B(H)$ be an algebraically $k$-quasi-*-class $A$ operator, and $\sigma(T)=\left\{\mu_{0}\right\}$, then $T-\mu_{0}$ is nilpotent.

Proof Assume $p(T)$ is $k$-quasi- $*$-class $A$ for some nonconstant polynomial $p(z)$. Since $\sigma(p(T))=p(\sigma(T))=\left\{p\left(\mu_{0}\right)\right\}$, the operator $p(T)-p\left(\mu_{0}\right)$ is nilpotent by Corollary 2.2. Let

$$
p(z)-p\left(\mu_{0}\right)=a\left(z-\mu_{0}\right)^{k_{0}}\left(z-\mu_{1}\right)^{k_{1}}\left(z-\mu_{t}\right)^{k_{t}},
$$

where $\mu_{j} \neq \mu_{s}$ for $j \neq s$. Then

$$
0=\left\{p(T)-p\left(\mu_{0}\right)\right\}^{m}=a^{m}\left(T-\mu_{0}\right)^{m k_{0}}\left(T-\mu_{1}\right)^{m k_{1}}\left(T-\mu_{t}\right)^{m k_{t}}
$$

and hence $\left(T-\mu_{0}\right)^{m k_{0}}=0$.

In the following theorem, we will prove that an algebraically $k$-quasi-*-class $A$ operator is polaroid.

Theorem 2.2 Let $T$ be an algebraically $k$-quasi-*-class $A$ operator. Then $T$ is polaroid.

Proof If $T$ is an algebraically $k$-quasi-*-class $A$ operator, then $p(T)$ is a $k$-quasi-*-class $A$ operator for some nonconstant polynomial $p$. Let $\mu \in \operatorname{iso}(\sigma(T))$, and let $E_{\mu}$ be the Riesz idempotent associated to $\mu$ defined by

$$
E:=\frac{1}{2 \pi i} \int_{\partial D}(\mu I-T)^{-1} d \mu
$$

where $D$ is a closed disk centered at $\mu$ which contains no other points of the spectrum of $T$. Then $T$ can be represented as follows:

$$
\left(\begin{array}{cc}
T_{1} & 0 \\
0 & T_{2}
\end{array}\right),
$$

where $\sigma\left(T_{1}\right)=\{\mu\}$ and $\sigma\left(T_{2}\right)=\sigma\left(T_{2}\right) \backslash\{\mu\}$. Since $T_{1}$ is algebraically $k$-quasi-*-class $A$ operator by Lemma 2.3 and $\sigma\left(T_{1}\right)=\{\mu\}$, it follows from Lemma 2.3 that $T_{1}-\lambda I$ is nilpotent. Therefore, $T_{1}-\mu$ has finite ascent and descent. On the other hand, since $T_{2}-\mu I$ is invertible, it has finite ascent and descent. Therefore, $T-\mu I$ has finite ascent and descent. Therefore, $\mu$ is a pole of the resolvent of $T$. Now if $\mu \in \operatorname{iso}(\sigma(T))$, then $\mu \in \pi(T)$. Thus, iso $(\sigma(T)) \in \pi(T)$, where $\pi(T)$ denotes the set of poles of the resolvent of $T$. Hence, $T$ is polaroid.

Recall that an operator $T$ is said to be hereditarily polaroid if every part of it is polaroid. Hence, it follows from Lemma 2.2 that a $k$-quasi- $*$-class $A$ operator is hereditarily polaroid

Corollary 2.3 A k-quasi-*-class A operator is isoloid. 


\section{Weyl-type theorems}

Let $X$ be a complex Banach space. For every $T \in B(X)$, define

$$
E(T):=\{\lambda \in \operatorname{iso} \sigma(T): 0<\alpha(\lambda I-T)\}
$$

and

$$
E^{a}(T):=\left\{\lambda \in \operatorname{iso} \sigma_{\mathrm{a}}(T): 0<\alpha(\lambda I-T)\right\} .
$$

Obviously, $E^{0}(T) \subseteq E(T) \subseteq E^{a}(T)$ for every $T \in L(X)$. Define

$$
\pi_{00}(T):=\{\lambda \in \operatorname{iso} \sigma(T): 0<\alpha(\lambda I-T)<\infty\}
$$

and

$$
\pi_{00}^{a}(T):=\left\{\lambda \in \text { iso } \sigma_{\mathrm{a}}(T): 0<\alpha(\lambda I-T)<\infty\right\} .
$$

Let $p_{00}(T):=\sigma(T) \backslash \sigma_{\mathrm{b}}(T)$, i.e., $p_{00}(T)$ is the set of all poles of the resolvent of $T$.

Definition 3.1 A bounded operator $T \in B(X)$ is said to satisfy Weyl's theorem, in symbol (W), if $\sigma(T) \backslash \sigma_{\mathrm{w}}(T)=\pi_{00}(T)$. $T$ is said to satisfy a-Weyl's theorem, in symbol (aW), if $\sigma_{\mathrm{a}}(T) \backslash \sigma_{\mathrm{uw}}(T)=\pi_{00}^{a}(T) . T$ is said to satisfy the property $(w)$, if $\sigma_{\mathrm{a}}(T) \backslash \sigma_{\mathrm{uw}}(T)=\pi_{00}(T)$.

Either $a$-Weyl's theorem or the property $(w)$ entails Weyl's theorem. The property $(w)$ and $a$-Weyl's theorem are independent; see [11].

The concept of semi-Fredholm operators has been generalized by Berkani $[19,20]$ in the following way: for every $T \in B(X)$ and a nonnegative integer $n$, let us denote by $T_{[n]}$ the restriction of $T$ to $T^{n}(X)$ viewed as a map from the space $T^{n}(X)$ into itself (we set $T_{[0]}=T$ ). $T \in B(X)$ is said to be semi-B-Fredholm (resp. B-Fredholm, upper semi-B-Fredholm, lower semi-B-Fredholm,) if for some integer $n \geq 0$, the range $T^{n}(X)$ is closed and $T_{[n]}$ is a semiFredholm operator (resp. Fredholm, upper semi-Fredholm, lower semi-Fredholm). In this case $T_{[m]}$ is a semi-Fredholm operator for all $m \geq n$ [20]. This enables one to define the index of a semi- $B$-Fredholm as ind $T=\operatorname{ind} T_{[n]}$. A bounded operator $T \in B(X)$ is said to be $B$-Weyl (respectively, upper semi-B-Weyl, lower semi-B-Weyl) if for some integer $n \geq 0$, $T^{n}(X)$ is closed and $T_{[n]}$ is Weyl (respectively, upper semi-Weyl, lower semi-Weyl). In an obvious way, all the classes of operators generate spectra, for instance, the $B$-Weyl spectrum $\sigma_{\mathrm{bw}}(T)$ and the upper $B$-Weyl spectrum $\sigma_{\mathrm{ubw}}(T)$. Analogously, a bounded operator $T \in B(X)$ is said to be $B$-Browder (respectively, upper semi-B-Browder, lower semi$B$-Browder) if for some integer $n \geq 0, T^{n}(X)$ is closed and $T_{[n]}$ is Weyl (respectively, upper semi-Browder, lower semi-Browder). The B-Browder spectrum is denoted by $\sigma_{\mathrm{bb}}(T)$, the upper semi-B-Browder spectrum by $\sigma_{\mathrm{ubb}}(T)$.

The generalized versions of Weyl-type theorems are defined as follows.

Definition 3.2 A bounded operator $T \in B(X)$ is said to satisfy generalized Weyl's theorem, in symbol, (gW), if $\sigma(T) \backslash \sigma_{\mathrm{bw}}(T)=E(T) . T \in B(X)$ is said to satisfy generalized a-Weyl's theorem, in symbol, (gaW), if $\sigma_{\mathrm{a}}(T) \backslash \sigma_{\mathrm{ubw}}(T)=E^{a}(T)$. $T \in L(X)$ is said to satisfy the generalized property $(w)$, in symbol, $(g w)$, if $\sigma_{\mathrm{a}}(T) \backslash \sigma_{\mathrm{ubw}}(T)=E(T)$. 
In the following diagrams, we resume the relationships between all Weyl-type theorems:

$$
\begin{aligned}
& (g w) \Rightarrow(w) \Rightarrow(\mathrm{W}), \\
& (\mathrm{gaW}) \Rightarrow(\mathrm{aW}) \Rightarrow(\mathrm{W}) ;
\end{aligned}
$$

see [21, Theorem 2.3], [11] and [22]. The generalized property $(w)$ and generalized $a$-Weyl's theorem are also independent; see [21]. Furthermore,

$$
\begin{aligned}
& (g w) \Rightarrow(\mathrm{gW}) \Rightarrow(\mathrm{W}), \\
& (\mathrm{gaW}) \Rightarrow(\mathrm{gW}) \Rightarrow(\mathrm{W}) ;
\end{aligned}
$$

see [21] and [22]. The converse of all these implications in general does not hold. Furthermore, by [23, Theorem 3.1],

$(W)$ holds for $T \Leftrightarrow$ Browder's theorem holds for $T$ and $p_{00}(T)=\pi_{00}(T)$.

Let $d_{A, B} \in B(H)$ denote either the generalized derivation $\delta_{A, B}=L_{A}-R_{B}$ or the elementary operator $\Delta_{A, B}=L_{A} R_{B}-I$, where $L_{A}$ and $R_{B}$ are the left and right multiplication operators defined on $B(H)$ by $L_{A}=A X$ and $R_{B}=X B$ respectively. We will show that if $A, B^{*}$ are $k$ quasi-*-class $A$, then $d_{A, B}$ is polaroid and satisfies all Weyl-type theorems. For this we need the following lemmas.

Lemma 3.1 [24] Let $A, B \in B(H)$. If $A, B$ are polaroid operators, then $d_{A, B}$ is polaroid.

Lemma 3.2 If $A, B^{*}$ are $k$-quasi-*-class $A$ operators, then $d_{A, B}$ is polaroid.

Proof It is known in a Hilbert space [14] that $B$ is polaroid if and only if $B^{*}$ is polaroid. Hence, it suffices to apply the previous lemma.

Recall that an operator $T \in B(X)$ is said to have the property $(\delta)$ if for every open covering $(U, V)$ of $\mathbb{C}$, we have $X=\mathcal{H}_{T}(\bar{U})+\mathcal{H}_{T}(\bar{V})$.

Lemma 3.3 Let $A, B \in B(H)$. If $A, B$ have the property $(\beta)$, then $d_{A, B}$ has SVEP.

Proof It is known [15, Theorem 2.5.5] that $B$ satisfies the property $(\beta)$ if and only if $B^{*}$ satisfies the property $(\delta)$. Since $A, B$ have the property $(\beta)$ by Theorem $2.1, B^{*}$ satisfies the property $(\delta)$. Hence, it results from [15, Corollary 3.6.16] that both $L_{A}$ and $R_{B}$ satisfy the Dunford property $(C)$. Since $L_{A}$ and $R_{B}$ commute, hence $L_{A}-R_{B}$ and $L_{A} R_{B}$ have SVEP by [15, Theorem 3.6.3 and Note 3.6.19]. Therefore, $d_{A, B}$ satisfies SVEP.

Corollary 3.1 Let $A, B \in B(H)$. If $A, B^{*}$ are $k$-quasi-*-class $A$ operators, then $d_{A, B}$ has SVEP.

If a Banach space operator $T$ has SVEP (everywhere), the single-valued extension property, then $T$ and $T^{*}$ satisfy Browder's (equivalently, generalized Browder's) theorem and $a$-Browder's (equivalently, generalized $a$-Browder's) theorem. A sufficient condition for an operator $T$ satisfying Browder's (generalized Browder's) theorem to satisfy Weyl's (resp. 
generalized Weyl's) theorem is that $T$ is polaroid. Now since $T$ and $T^{*}$ are polaroid operators when $T$ is algebraically $k$-quasi-*-class $A$, then Weyl's theorem and generalized Weyl's theorem hold for $T$ and $T^{*}$ when $T$ is algebraically $k$-quasi-*-class $A$. Now, observe for polaroid operators $T$ satisfying generalized Weyl's theorem,

$$
E(T)=\pi(T)=\pi\left(T^{*}\right)=E\left(T^{*}\right),
$$

where $\pi(T)$ is the set of poles of the resolvent of $T$. Hence, for a polaroid operator $T, T^{*}$ satisfies generalized Weyl's theorem if and only if $T$ satisfies generalized Weyl's theorem if and only if $T$ satisfies Weyl's theorem if and only if $T^{*}$ satisfies Weyl's theorem.

Theorem 3.1 Let $T, A, B \in B(H)$. If $T, A, B^{*}$ are algebraically $k$-quasi-*-class $A$, then the following statements are equivalent.

(i) generalized Weyl's theorem holds for $T^{*}$ (resp. for $d_{A^{*}, B^{*}}$ ).

(ii) generalized Weyl's theorem holds for $T$ (resp. for $d_{A, B}$ ).

(iii) Weyl's theorem holds for $T$ (resp. for $d_{A, B}$ ).

Recall that a sufficient condition for an operator $T$ satisfying Browder's (generalized Browder's) theorem to satisfy Weyl's (resp. generalized Weyl's) theorem is that $T$ is polaroid. Observe that if $T \in B(H)$ has SVEP, then $\sigma(T)=\overline{\sigma_{a}\left(T^{*}\right)}$. Hence, if $T$ has SVEP and is polaroid, then $T^{*}$ satisfies generalized $a$-Weyl's (so, also $a$-Weyl's) theorem [14]. It follows from Theorem 2.1 that $k$-quasi- $*$-class $A$ operator has SVEP. Thus, we have the following theorem.

Theorem 3.2 Let $T, A, B \in B(H)$.

(i) If $T$ is algebraically $k$-quasi-*-class $A$ and $A, B^{*}$ are $k$-quasi-*-class $A$, then generalized a-Weyl's theorem holds for $T^{*}$ (resp. for $d_{A^{*}, B^{*}}$ ).

(ii) If $T^{*}$ is algebraically $k$-quasi-*-class $A$ and $A, B^{*}$ are $k$-quasi-*-class $A$, then generalized a-Weyl's theorem holds for $T$ (resp. for $d_{A^{*}, B^{*}}$ ).

Recall [14] that if $T$ is polaroid, then $T$ satisfies generalized Weyl's theorem (resp. generalized $a$-Weyl's theorem) if and only if $T$ satisfies Weyl's theorem (resp. $a$-Weyl's theorem). Hence if $T$ is an algebraically $k$-quasi-*-class $A$ operator, we have the following result.

Theorem 3.3 Let $T, A, B \in B(H)$. If $T$ is algebraically $k$-quasi-*-class $A$ and $A, B^{*}$ are k-quasi-*-class $A$, then

(i) Weyl's theorem holds for $T$ (resp. for $d_{A, B}$ ) if and only if generalized Weyl's theorem holds for $T$ (resp. for $d_{A, B}$ ).

(ii) a-Weyl's theorem holds for $T^{*}$ (resp. for $\left.d_{A^{*}, B^{*}}\right)$ if and only if generalized a-Weyl's theorem holds for $T^{*}$ (resp. for $d_{A^{*}, B^{*}}$ ).

Theorem 3.4 Let $T, A, B \in B(H)$.

(i) If $T^{*}$ is algebraically $k$-quasi-*-class $A$ and $A, B^{*}$ are $k$-quasi-*-class $A$, then Weyl's theorem, a-Weyl's theorem, generalized Weyl's theorem and generalized $a$-Weyl's theorem hold for $T$ (resp. $\left.d_{A, B}\right)$ and these are equivalent.

(ii) If $T$ is algebraically $k$-quasi-*-class $A$ and $A, B^{*}$ are $k$-quasi-*-class $A$, then Weyl's theorem, $a$-Weyl's theorem, generalized Weyl's theorem and generalized a-Weyl's theorem hold for $T^{*}\left(\right.$ resp. $\left.d_{A^{*}, B^{*}}\right)$ and are equivalent. 
Proof Since Weyl's theorem holds for $T$ (resp. for $d_{A, B}$ ). It suffices to show that Weyl's theorem is equivalent to each one of the other Weyl-type theorems for $T$ (resp. for $d_{A, B}$ ), generalized or not. Since $T^{*}$ (resp. $d_{A^{*}, B^{*}}$ ) has SVEP, Weyl's theorem and $a$-Weyl's theorem hold for $T$ (resp. for $d_{A, B}$ ) and are equivalent by [8, Theorem 2.16]. Theorem 3.3(i) implies that Weyl's theorem and generalized Weyl's theorem hold for $T$ (resp. for $d_{A, B}$ ) and are equivalent. Now $a$-Weyl's theorem and generalized $a$-Weyl's theorem hold for $T$ (resp. for $\left.d_{A, B}\right)$ and are equivalent by Theorem 3.3(ii).

Let $f \in \operatorname{Hol}(\sigma(T))$, where $\operatorname{Hol}(\sigma(T))$ is the space of all functions that are analytic in an open neighborhoods of $\sigma(T)$. If $T$ is polaroid, then $f(T)$ is polaroid too [14]. Thus, we have

Theorem 3.5 Let $T, A, B \in B(H)$.

(i) If $T^{*}$ is algebraically $k$-quasi-*-class $A$ and $A, B^{*}$ are $k$-quasi-*-class $A$, then $f\left(T^{*}\right)$ (resp. $\left.f\left(d_{A^{*}, B^{*}}\right)\right)$ satisfies Weyl's theorem, a-Weyl's theorem, generalized Weyl's theorem and generalized a-Weyl's theorem.

(ii) If $T$ is algebraically $k$-quasi-*-class $A$ and $A, B^{*}$ are $k$-quasi-*-class $A$, then $f(T)$ (resp. $\left.f\left(d_{A, B}\right)\right)$ satisfies Weyl's theorem, a-Weyl's theorem, generalized Weyl's theorem and generalized $a$-Weyl's theorem.

Proof

(i) If $T^{*}$ is algebraically $k$-quasi-*-class $A$ and $A, B^{*}$ are $k$-quasi-*-class $A$, then $T^{*}$ (resp. $d_{A^{*}, B^{*}}$ ) is polaroid [14]. Since $T^{*}$ (resp. $d_{A^{*}, B^{*}}$ ) is polaroid, the result holds by [14, Theorem 3.12]

(ii) If $T$ is algebraically $k$-quasi-*-class $A$ and $A, B^{*}$ are $k$-quasi-*-class $A$, then $f(T)$ (resp. $f\left(d_{A, B}\right)$ ) is polaroid. Since $T$ (resp. $f\left(d_{A, B}\right)$ ) is polaroid, the result holds by [14, Theorem 3.12].

According to [14, Theorem 3.12] Theorem 3.4 may be extended as follows.

Theorem 3.6 Let $T, A, B \in B(H)$.

(i) If $T^{*}$ is algebraically $k$-quasi-*-class $A$ and $A, B^{*}$ are $k$-quasi-*-class $A$, then Weyl's theorem, a-Weyl's theorem, generalized Weyl's theorem and generalized a-Weyl's theorem hold for $f(T)$ (resp. $f\left(d_{A, B}\right)$ ) and these are equivalent.

(ii) If $T$ is algebraically $k$-quasi-*-class $A$ and $A, B^{*}$ are $k$-quasi-*-class $A$, then Weyl's theorem, a-Weyl's theorem, generalized Weyl's theorem and generalized a-Weyl's theorem hold for $f\left(T^{*}\right)$ (resp. $f\left(d_{A^{*}, B^{*}}\right)$ ) and these are equivalent.

Remark 3.1 According to [14], the previous results on a Weyl-type theorem still true for the property $(w)$.

\footnotetext{
Competing interests

The author declares that they have no competing interests.
}

\section{Acknowledgements}

The author would like to thank the referee for his good reading of the paper and his comments. This paper is supported by Taibah University Research Center Project (1433-808). 


\section{References}

1. Arora, SC, Arora, P: On p-quasihyponormal operators for $0<p<1$. Yokohama Math. J. 41, $25-29$ (1993)

2. Furuta, T: Invitation to Linear Operators. From Matrices to Bounded Linear Operators in Hilbert space. Taylor \& Francis, London (2001)

3. Jean, $\mid \mathrm{H}$, Kim, $\mid \mathrm{H}$ : On operators satisfying $T^{*}\left|T^{2}\right| T \geq T^{*}|T|^{2} T$. Linear Algebra Appl. 418, 854-862 (2006)

4. Shen, JL, Zuo, F, Yang, CS: On operators satisfying $T^{*}\left|T^{2}\right| T \geq T^{*}\left|T^{* 2}\right| T$. Acta Math. Sin. Engl. Ser. 26(11), pp. $2109-2116$ (2010)

5. Mecheri, S: Spectral properties of k-quasi-*-class A operators. Studia Math. 208, 87-96 (2012)

6. Aiena, P: On the spectral properties of some classes of operators. In: RIMS Conference 1678: Prospect of Non-Commutative Analysis in Operator Theory, Kyoto University, pp. 5-21 (2009)

7. Curto, RE, Han, YM: Weyl's theorem for algebraically paranormal operators. Integral Equ. Oper. Theory 47, 307-314 (2003)

8. Aiena, P, Guillen, GR: Weyl's theorem for perturbations of paranormal operators. Proc. Am. Math. Soc. 35, 2433-2442 (2007)

9. Mecheri, S: Weyl's theorem for algebraically class A operators. Bull. Belg. Math. Soc. Simon Stevin 14, $239-246$ (2007)

10. Rakoc̃evič, V: On the essential approximate point spectrum II. Mat. Vesn. 36, 89-97 (1984)

11. Aiena, P, Peña, P: A variation on Weyl's theorem. J. Math. Anal. Appl. 324, 566-579 (2006)

12. Aiena, P, Guillen, J, Peña, P: Property $(w)$ for perturbation of polaroid operators. Linear Algebra Appl. 4284, 1791-1802 (2008)

13. Heuser, H: Functional Analysis. Dekker, New York (1982)

14. Aiena, P, Aponte, E, Balzan, E: Weyl type theorems for left and right Polaroid operators. Integral Equ. Oper. Theory 66 , $1-20(2010)$

15. Laursen, KB, Neumann, MM: An Introduction to Local Spectral Theory. London Mathematical Society Monographs. Oxford University Press, Oxford (2000)

16. Aiena, P: Fredholm and Local Spectral Theory with Applications to Multipliers. Kluwer Academic, Dordrecht (2004)

17. Rosenblum, MA: On the operator equation $B X-X A=Q$. Duke Math. J. 23, 263-269 (1956)

18. Yang, Y: On algebraically total *-paranormal. Nihonkai Math. J. 10, 187-194 (1999)

19. Berkani, M: On a class of quasi-Fredholm operators. Integral Equ. Oper. Theory 34(1), 244-249 (1999)

20. Berkani, M, Sarih, M: On semi B-Fredholm operators. Glasg. Math. J. 43, 457-465 (2001)

21. Berkani, M, Amouch, M: On the property ( $\mathrm{gw}$ ). Mediterr. J. Math. 5(3), 371-378 (2008)

22. Berkani, M, Koliha, JJ: Weyl type theorems for bounded linear operators. Acta Sci. Math. (Szeged) 69(1-2), 359-376 (2003)

23. Aiena, P: Classes of operators satisfying a-Weyl's theorem. Stud. Math. 169, 105-122 (2005)

24. Cho, M, Djordjevic, S, Duggal, B: Bishop's property $\beta$ and an elementary operator. Hokkaido Math. J. 40(3), $337-356$ (2011)

doi:10.1186/1029-242X-2012-244

Cite this article as: Mecheri: On operators satisfying an inequality. Journal of Inequalities and Applications 2012

2012:244

\section{Submit your manuscript to a SpringerOpen ${ }^{\odot}$ journal and benefit from:}

- Convenient online submission

- Rigorous peer review

- Immediate publication on acceptance

Open access: articles freely available online

- High visibility within the field

- Retaining the copyright to your article 